\section{Lymph node fibrosis after ischaemic injury}

Kidney fibrosis is a well-known consequence of repeated ischaemia-reperfusion injury (IRI), but what happens to the draining lymph node? Reza Abdi and his team now report that the kidney lymph node not only has an important role in lymphocyte activation after IRI but also undergoes fibrotic changes.

"Our early work showed that the kidney lymph node undergoes a massive microstructural transformation following IRI," remarks Abdi. As fibroblastic reticular cells (FRCs) have a key role in the maintenance of the lymph node structural framework, which facilitates the trafficking of immune cells and antigens, the researchers focused their recent study on the impact of IRI on FRCs and lymph node architecture.

Their new findings show that following kidney IRI, both T cells and FRCs actively proliferate in the draining lymph node. This proliferation is accompanied by increased collagen deposition, macrophage infiltration and expansion of high endothelial venules. Expression of the profibrotic genes encoding $\alpha$-smooth muscle actin and fibronectin also increases in the kidney lymph node after IRI.
Furthermore, the researchers demonstrate that in the setting of repeated IRI, both the kidney and lymph node become increasingly fibrotic as they accumulate collagen I and fibronectin. In contrast to the expansion observed in acute IRI, FRC density in the lymph node decreases following repeated IRI owing to senescence of these cells. In the acute setting, FRC depletion reduced T cell activation in the lymph node and kidney injury. However, in the chronic setting of recurrent IRI, transfer of exogenous FRCs, which homed to the lymph node draining the ischaemic kidney, ameliorated renal fibrosis and restored lymph node microarchitecture.

"I believe that the reason why we have not been able to target organ fibrosis effectively is that we have forgotten about the critical role of the draining lymph nodes in regulating organ inflammation," comments Abdi. "To control organ fibrosis we may need to target lymph node fibrosis at the same time."

Monica Wang

ORIGINAL ARTICLE Maarouf, O. H. et al. Repetitive ischemic injuries to the kidneys result in lymph node fibrosis and impaired healing. JCI Insight 3, e120546 (2018)

\title{
TRANSPLANTATION
}

\section{$M_{\text {regs }}$ kick-start transplant tolerance}

Cell-based immunosuppressive therapies hold great promise for preventing transplant rejection and are the subject of active research. Now, Riquelme et al. provide further insight into the role of regulatory macrophages $\left(M_{\text {regs }}\right)$ in controlling the immune response of kidney transplant recipients.

"Previous work demonstrated that allograft-infiltrating, monocyte-derived macrophages with immune regulatory functions are indispensable for establishing and maintaining transplantation tolerance in animal models," explains lead investigator James Hutchinson. The researchers generated $M_{\text {regs }}$ in vitro from $C D 14^{+}$blood monocytes to study their interactions with allogeneic $T$ cells. Co-culture of FOXP3- $3^{-} D 4^{+} T$ cells and $\mathrm{M}_{\text {regs }}$ demonstrated that $\mathrm{M}_{\text {regs }}$ induced the formation of IL-10-producing $\mathrm{FOXP}^{+}$regulatory $\mathrm{T}\left(\mathrm{T}_{\text {reg }}\right)$ cells, so-called $M_{\text {reg }}$-induced $\mathrm{T}_{\text {reg }}$ cells ( $\mathrm{miT}_{\text {reg }}$ cells); this effect required a direct interaction between the two cell types. Furthermore, using inhibitors, the researchers established that the $M_{\text {reg }}$-induced formation of $\mathrm{miT}_{\text {reg }}$ cells involves multiple non-redundant signalling pathways, including those mediated by indoleamine 2,3-dioxygenase (IDO), transforming growth factor- $\beta$ (TGF $\beta$ ), retinoic acid, Notch and progestagen-associated endometrial protein (PAEP; also known as glycodelin).

Transcriptional profiling of $\mathrm{miT}_{\text {reg }}$ cells revealed that these cells expressed higher levels of T cell immunoreceptor with immunoglobulin and ITIM domains (TIGIT; a negative regulator of T cell-mediated immunity) than non-induced $\mathrm{T}_{\text {reg }}$ cells. Importantly, adoptive transfer of allogeneic $M_{\text {regs }}$ to patients prior to living donor kidney transplantation led to an enrichment of $\mathrm{T}_{\text {reg }}$ cells phenotypically similar to $\mathrm{mit}_{\text {reg }}$ cells.

" $M_{\text {regs }}$ seem to elicit a TIGIT $^{+} T_{\text {reg }}$ cell response after adoptive transfer, which might sustain transplant-protective regulation beyond the lifespan of transferred $\mathrm{M}_{\text {regs, }}$, says Hutchinson.

Grant Otto

ORIGINAL ARTICLE Riquelme, P. et al. TIGIT+ ${ }^{+}$Tregs elicited by human regulatory macrophages control T cell immunity. Nat. Commun. 9, 2858 (2018)

\section{GLP1 fragments protect the kidney}

In contrast to other incretin-based therapies, glucagon-like peptide 1 receptor (GLP1R) agonists with close peptide homology to GLP1 have been shown to improve renal and cardiovascular outcomes in patients with type 2 diabetes mellitus (T2DM). Now, Michael Lehrke, Peter Boor and colleagues report that GLP1 fragments have renoprotective effects.

Binding of GLP1 to GLP1R stimulates insulin production, slows gastric emptying and reduces appetite. However, GLP1 is rapidly inactivated by dipeptidyl peptidase 4 (DPP4) and cleaved into smaller fragments by neutral endopeptidase. As these fragments cannot activate GLP1R, DPP4 inhibitors and GLP1R agonists are effective therapies to lower glucose levels in patients with T2DM.

"The remarkable differences in outcomes in diabetic patients treated with different incretin-based therapies led us to hypothesize that GLP1 cleavage products have relevant biological activities independent of GLP1R," says Lehrke. To investigate these activities the researchers overexpressed GLP1 constructs in $d b / d b$ diabetic mice. They report that only overexpression of DPP4-resistant full-length GLP1 increased glucose tolerance; however, overexpression of either DPP4-resistant GLP1 or GLP1 cleavage fragments reduced kidney tubular damage in these mice.

"The renoprotective effects were glucose-independent and seemed to be mediated by immune modulation rather than by a direct effect on the kidneys," remarks Boor. In fact, the GLP1 constructs had no effect on albuminuria or electrolyte excretion but markedly decreased the renal myeloid and $T$ cell infiltrate, reduced the cardiac T cell infiltrate and restored the frequency of circulating $T$ cells to normal levels.

Pretreatment with GLP1, GLP1 cleavage products or the GLP1R agonist liraglutide, which mimics the peptide structure of GLP1, had similar immunomodulatory effects in a mouse model of ischaemia-reperfusion injury.

"We now plan to focus on the downstream pathways that mediate the organ-protective and immunomodulatory effects of GLP1 cleavage products and on their therapeutic potential in various diseases," says Boor.

Monica Wang

ORIGINAL ARTICLE Moellmann, J. et al. Glucagon-like peptide- 1 and its cleavage products are renoprotective in murine diabetic nephropathy. Diabetes https://doi.org/ 10.2337/db17-1212 (2018) 